\title{
Using Cloudy Kernels for Imprecise Linear Filtering
}

\author{
Sebastien Destercke ${ }^{1}$ and Olivier Strauss ${ }^{2}$ \\ 1 INRA/CIRAD, UMR1208, 2 place P. Viala, F-34060 Montpellier cedex 1, France \\ 2 LIRMM (CNRS \& Univ. Montpellier II), 161 rue Ada, F-34392 Montpellier cedex 5, France \\ sebastien.destercke@supagro.inra.fr, olivier.strauss@lirmm. fr
}

\begin{abstract}
Selecting a particular summative (i.e., formally equivalent to a probability distribution) kernel when filtering a digital signal can be a difficult task. To circumvent this difficulty, one can work with maxitive (i.e., formally equivalent to a possibility distribution) kernels. These kernels allow to consider at once sets of summative kernels with upper bounded bandwith. They also allow to perform a robustness analysis without additional computational cost. However, one of the drawbacks of filtering with maxitive kernels is sometimes an overly imprecise output, due to the limited expressiveness of summative kernels. We propose to use a new uncertainty representation, namely cloud, to achieve a compromise between summative and maxitive kernels, avoiding some of their respective shortcomings. The proposal is then experimented on a simulated signal.
\end{abstract}

Keywords: Signal treatment, interval-valued fuzzy sets, generalised p-boxes.

\section{Introduction}

Reconstructing a continuous signal from a set of sampled and possibly corrupted observations is a common problem in both digital analysis and signal processing [1]. In this context, kernel-based methods can be used for different purposes: reconstruction, impulse response modelling, interpolation, (non)-linear transformations, filtering, etc.

Most kernels used in signal processing are linear combination of summative kernels, which are positive functions with an integral equal to one. A summative kernel can therefore be associated to a particular probability distribution. Still, how to choose a particular kernel and its parameters to filter a given signal is often a tricky question. Using maxitive kernels [2], that is kernels that are formally equivalent to possibility distributions [3], can overcome this difficulty. This can be done by interpreting maxitive kernels and associated possibility distributions [3] as sets of summative kernels (or sets of probability distributions [4]). The output of a maxitive kernel-based filtering is an interval valued signal that gathers all the outputs of conventional filtering based on the summative kernels belonging to the considered set. This property allows to perform a rosbustness or sensitivity analysis of the filtering during the filtering process itself.

The main interests of maxitive kernels are their simplicity of representation and their computational tractability. The price to pay for such features is a limited expressiveness and the impossibility to exclude unwanted summative kernels from the set represented by maxitive kernels in some applications. For instance, this set always includes a Dirac measure, meaning that the filtered interval-valued signal always includes the original (noisy) signal itself. 
To overcome this shortcoming of maxitive kernel while keeping their interesting features, we propose to use another uncertainty representation, called clouds [5], as a compromise between summative and maxitive kernels. we call the resulting kernels cloudy kernels. The interest of cloudy kernels is two-fold: they are more expressive than maxitive kernels, the latter being a special case of the former [6], and their use only require low computational efforts, an important feature in signal processing.

We first introduce summative and maxitive kernels, before showing how cloudy kernels can act as a compromise between the two (Section 2). The computational aspects of using cloudy kernels are then discussed, and an efficient algorithm to perform signal filtering with them is devised (Section 3). Some experiments on a simulated signal are then performed and their results discussed (Section 4).

\section{Between Summative and Maxitive Kernels: Cloudy Kernels}

This section recalls the basics of summative and maxitive kernels. It then introduces cloudy kernels and shows how they can model summative kernels with lower-bounded bandwidth. For readability purpose, we will restrict ourselves to representations defined on the real line $\mathbb{R}$ and its discretization $\mathscr{X} 1$

\subsection{Summative Kernels}

A summative kernel $\kappa$ is formally equivalent to a Lebesgue-measurable probability distribution $\kappa: \mathbb{R} \rightarrow \mathbb{R}^{+}$, and can be interpreted as such. The associated probability measure $P_{K}: \mathscr{B} \rightarrow[0,1]$ defined on the real Borel agebra $\mathscr{B}$ is such that, for any measurable subset $A \subseteq \mathbb{R}$ (also called an event), $P_{\kappa}(A)=\int_{A} \kappa(x) d x$.

In this paper, we restrict ourselves to bounded, symmetrical and mono-modal kernels. To shorten notations, we consider that kernels belong to a family of kernels parameterized by their bandwidth $\Delta$ and defined on a compact interval $[-\Delta, \Delta] \subseteq \mathbb{R}$ centred around zero. Typical kernels belonging to such families are recalled and represented in Table 1. We denote them by $\kappa_{\Delta}$, and they are such that $\kappa_{\Delta}(x)=\kappa_{\Delta}(-x)$. To a summative kernel $\kappa_{\Delta}$ can be associated its cumulative distribution function $F_{\kappa_{\Delta}}:[-\Delta, \Delta] \rightarrow[0,1]$ such that, for any $x \in[-\Delta, \Delta], F_{\kappa_{\Delta}}(x)=\int_{-\Delta}^{x} \kappa_{\Delta}(x) d x$ which is such that $F_{\kappa_{\Delta}}(0)=1 / 2$ and $F_{\kappa_{\Delta}}(x)+F_{\kappa_{\Delta}}(-x)=1$.

\subsection{Maxitive Kernels}

A maxitive kernel $\pi$ is a normalised function $\pi: \mathbb{R} \rightarrow[0,1]$ with at least one $x \in \mathbb{R}$ such that $\pi(x)=1$. A maxitive kernel can be associated to a possibility distribution [3], hence inducing two (lower and upper) confidence measures, respectively called necessity and possibility measures. They are such that, for any event $A \subseteq \mathbb{R}$, we have:

$$
\Pi(A)=\max _{x \in A} \pi(x) \quad N(A)=1-\Pi\left(A^{c}\right)=\inf _{x \in A^{c}}(1-\pi(x)),
$$

\footnotetext{
${ }^{1}$ Extension of presented methods to some product space $\mathbb{R}^{p}$ is straightforward.
} 
Table 1. Some classical summative kernels

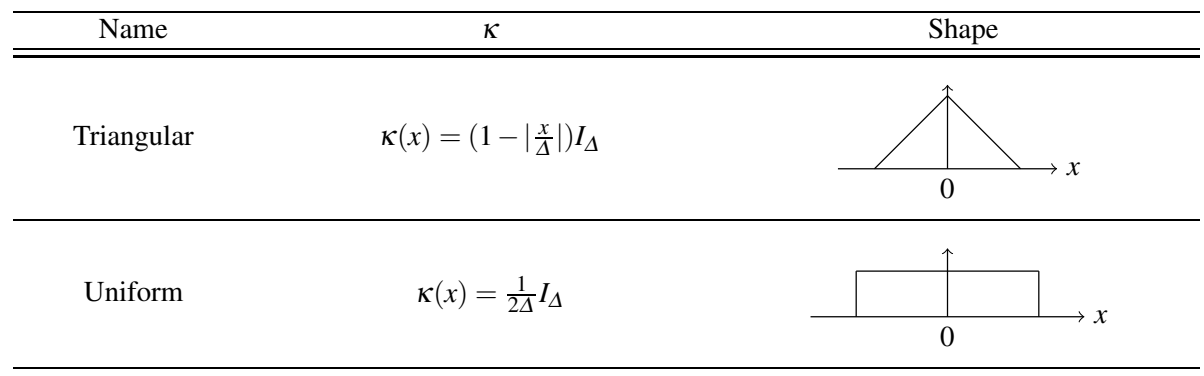

with $A^{c}$ the complement of $A$. A maxitive kernel $\pi$ can be associated to a set of summative kernels $\mathscr{P}_{\pi}$ dominated by the possibility measure $\Pi$ of $\pi$, such that $\mathscr{P}_{\pi}=$ $\left\{\kappa \in \mathbb{P}_{\mathbb{R}} \mid \forall A \subseteq \mathbb{R}, P(A) \leq \Pi(A)\right\}$, with $\mathbb{P}_{\mathbb{R}}$ the set of all summative kernels over $\mathbb{R}$. If a summative kernel $\kappa$ is in $\mathscr{P}_{\pi}$, we say, by a small abuse of language, that $\pi$ includes $\kappa$. This interpretation makes maxitive kernels instrumental tools to filter signal when the identification of a single summative kernel is difficult.

There are many ways to build a maxitive kernel including a given summative kernel [7]. Here, we consider the so-called Dubois-Prade transformation, since it provides the most specific solution. Given a summative kernel $\kappa_{\Delta}$, the maxitive kernel $\pi_{\kappa_{\Delta}}$ resulting from the Dubois-Prade transformation is such that

$$
\pi_{\kappa_{\Delta}}(x)=\left\{\begin{array}{cc}
2 * F_{\kappa_{\Delta}}(x) & \text { if } x \leq 0 \\
2 *\left(1-F_{\kappa_{\Delta}}(x)\right) & \text { if } x>0
\end{array}\right.
$$

We will denote by $\pi_{\kappa_{\Delta}}^{+}, \pi_{\kappa_{\Delta}}^{-}$the following functions

$$
\pi_{\kappa_{\Delta}}^{-}(x)=\left\{\begin{array}{cl}
\pi_{\kappa_{\Delta}}(x) & \text { if } x \leq 0 \\
1 & \text { if } x>0
\end{array} \quad \pi_{\kappa_{\Delta}}^{+}(x)=\left\{\begin{array}{cc}
1 & \text { if } x \leq 0 \\
\pi_{\kappa_{\Delta}}(x) & \text { if } x>0
\end{array}\right.\right.
$$

The (convex) set $\mathscr{P}_{\pi_{\kappa_{\Delta}}}$ includes, among others, all summative kernels $\kappa_{\Delta^{\prime}}$ with $\Delta^{\prime} \in$ $[0, \Delta]$ [7]. Hence, maxitive kernels allow to consider families of kernels whose bandwidth are upper-bounded, but not lower-bounded, which in some situations may be a shortcoming. For instance, in those cases where it is desirable to smoothen a signal, the interval-valued signal resulting from an imprecise filtering should not envelope the initial signal, i.e., the Dirac measure should be excluded from the set of summative kernels used to filter. It is therefore desirable to dispose of representations allowing to model sets of summative kernels whose bandwidths are both lower- and upper-bounded. Next sections show that the uncertainty representation called clouds can meet such a need.

\subsection{Cloudy Kernels}

Clouds, the uncertainty representation used to model cloudy kernels, have been introduced by Neumaier [5]. On the real line, they are defined as follows: 
Definition 1. A cloud is a pair of mappings $[\pi, \eta]$ from $\mathbb{R}$ to the unit interval $[0,1]$ such that $\eta \leq \pi$ and there is at least one element $x \in \mathbb{R}$ such that $\pi(x)=1$ and one element $y \in \mathbb{R}$ such that $\eta(y)=0$

A cloud $[\pi, \eta]$ induces a probability family $\mathscr{P}_{[\pi, \eta]}$ such that

$$
\mathscr{P}_{[\pi, \eta]}=\left\{\kappa \in \mathbb{P}_{\mathbb{R}} \mid P_{\kappa}(\{x \mid \eta(x) \geq \alpha\}) \leq 1-\alpha \leq P_{\kappa}(\{x \mid \pi(x)>\alpha\})\right\} .
$$

And $\mathscr{P}_{[\pi, \eta]}$ induces lower and upper confidence measures $\underline{P}_{[\pi, \eta]}, \bar{P}_{[\pi, \eta]}$ such that, for any event $A \subseteq \mathbb{R}, \underline{P}_{[\pi, \eta]}(A)=\inf _{\kappa \in \mathscr{P}_{[\pi, \eta]}} P_{\kappa}(A)$ and $\bar{P}_{[\pi, \eta]}(A)=\sup _{\kappa \in \mathscr{P}_{[\pi, \eta]}} P_{\kappa}(A)$. Also note that, formally, clouds are equivalent to interval-valued fuzzy sets having boundary conditions (i.e., $\pi(x)=1$ and $\eta(y)=0$ for some $(x, y) \in \mathbb{R}^{2}$ ). A family of clouds that will be of particular interest here are the comonotonic clouds [6]. They are defined as follows:

Definition 2. A cloud is comonotonic if $\forall x, y \in \mathbb{R}, \pi(x)<\pi(y) \Rightarrow \eta(x) \leq \eta(y)$

A cloudy kernel is simply a pair of functions $[\pi, \eta]$ that satisfies Definition 1 As for maxitive kernels, we can associate $\mathscr{P}_{[\pi, \eta]}$ to the corresponding set of summative kernels. In this paper, we will restrict ourselves to cloudy kernels induced by bounded, symmetric and unimodal comonotonic clouds. Again, to make notations easier, we will consider that they are defined on the interval $[-\Delta, \Delta]$.

Definition 3. A unimodal symmetric cloudy kernel defined on $[-\Delta, \Delta]$ is such that, for any $x \in[-\Delta, \Delta], \eta(x)=\eta(-x), \pi(x)=\pi(-x)$ and $\eta, \pi$ are non-decreasing (nonincreasing) in $[-\Delta, 0]([0, \Delta])$

As for maxitive kernels, given a unimodal symmetric cloudy kernel, we will denote by $\eta^{+}, \eta^{-}$the functions such that

$$
\eta^{-}(x)=\left\{\begin{array}{cl}
\eta(x) & \text { if } x \leq 0 \\
1 & \text { if } x>0
\end{array} \quad \eta^{+}(x)=\left\{\begin{array}{cc}
1 & \text { if } x \leq 0 \\
\eta(x) & \text { if } x>0
\end{array}\right.\right.
$$

Two particular cases of comonotonic symmetric cloudy kernel are the so-called thin and fuzzy clouds. A cloudy kernel is said to be thin if $\forall x \in \mathbb{R}, \pi(x)=\eta(x)$, i.e., if the two mappings coincide. A cloudy kernel is said to be fuzzy if $\forall x \in \mathbb{R}, \eta(x)=0$, i.e. if the lower mapping $\eta$ conveys no information.

A cloudy kernel is pictured in Figure 1 Note that a fuzzy cloudy kernel $[\pi, \eta]$ induces the same summative kernel set $\mathscr{P}_{[\pi, \eta]}$ as the maxitive kernel $\pi$. We now recall some useful properties of clouds and cloudy kernels.

Proposition 1. A cloudy kernel $[\pi, \eta]$ is included in another one $\left[\pi^{\prime}, \eta^{\prime}\right]$ (in the sense that $\left.\mathscr{P}_{[\pi, \eta]} \subseteq \mathscr{P}_{\left[\pi^{\prime}, \eta^{\prime}\right]}\right)$ if and only if, for all $x \in \mathbb{R},[\pi(x), \eta(x)] \subseteq\left[\pi^{\prime}(x), \eta^{\prime}(x)\right]$.

Hence, given a cloudy kernel $[\pi, \eta]$, any thin cloud $\left[\pi^{\prime}, \eta^{\prime}\right]$ such that $\eta \leq \eta^{\prime}=\pi^{\prime} \leq \pi$ is included in $[\pi, \eta]$. Inversely, for any thin cloud $\left[\pi^{\prime}, \eta^{\prime}\right]$ not satisfying this condition (i.e. $\exists x$ such that $\eta^{\prime}(x)<\eta(x)$ or $\pi^{\prime}(x)>\pi(x)$ ), we have $\mathscr{P}_{[\pi, \eta]} \cap \mathscr{P}_{\left[\pi^{\prime}, \eta^{\prime}\right]}=\emptyset$.

Proposition 2. The convex set $\mathscr{P}_{[\pi, \eta]}$ induced by a thin cloud $[\pi, \eta]$ includes the two summative kernels having for cumulative distributions $F^{-}, F^{+}$such that, for all $x \in \mathbb{R}$

$$
F^{-}(x)=\eta^{-}(x)=\pi^{-}(x) \quad ; \quad F^{+}(x)=1-\eta^{+}(x)=1-\pi^{+}(x) .
$$

$\mathscr{P}_{[\pi, \eta]}$ being a convex set, any convex combination of $F^{-}, F^{+}$is also in the thin cloud. 


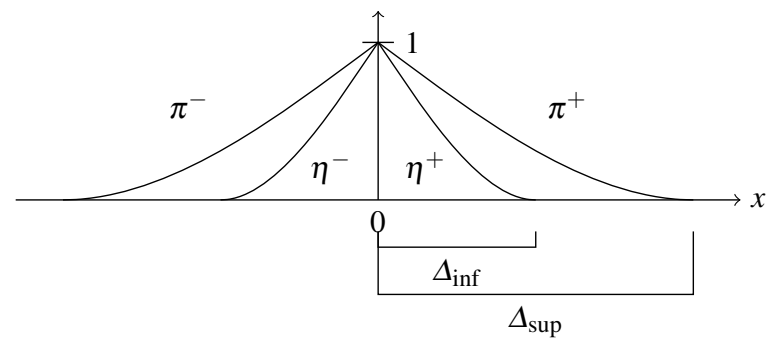

Fig. 1. Example of cloudy kernel

\subsection{Summative Kernel Approximation with Cloudy Kernels}

Let us show that cloudy kernels can remediate to the main drawback of maxitive kernels, i.e. they can model sets of summative kernels $\kappa_{\Delta}$ where $\Delta$ is lower and upperbounded. Assume that we want to represent the set of summative kernels $\kappa_{\Delta}$ such that $\Delta \in\left[\Delta_{\text {inf }}, \Delta_{\text {sup }}\right]$. To satisfy this requirement, we propose to consider the cloudy kernel $[\pi, \eta]_{\left[\Delta_{\text {inf }}, \Delta_{\text {sup }}\right]}$ such that, for any $x \in \mathbb{R}$ :

$$
\pi_{\Delta_{\text {sup }}}(x)=\left\{\begin{array}{cc}
2 * F_{\Delta_{\text {sup }}}(x) & \text { if } x \leq 0 \\
2 *\left(1-F_{\Delta_{\text {sup }}}(x)\right) & \text { if } x \geq 0
\end{array} ; \eta_{\Delta_{\text {inf }}}(x)=\left\{\begin{array}{cc}
2 * F_{\Delta_{\text {inf }}}(x) & \text { if } x \leq 0 \\
2 *\left(1-F_{\Delta_{\text {inf }}}(x)\right) & \text { if } x \geq 0
\end{array}\right.\right.
$$

Let us first show that this cloud contains all the desired summative kernels, starting with the summative kernels such that $\Delta=\Delta_{\text {inf }}$ and $\Delta=\Delta_{\text {sup }}$.

Proposition 3. The cloudy kernel $[\pi, \eta]_{\left[\Delta_{\text {iff }}, \Delta_{\text {sup }}\right]}$ includes the two summative kernels $\kappa_{\Delta_{\text {inf }}}$ and $\kappa_{\Delta_{\text {sup }}}$ having for cumulative distributions $F_{\Delta_{\text {inf }}}, F_{\Delta_{\text {sup }}}$.

Proof. From the definition of our cloudy kernel, we have that the thin cloudy kernels

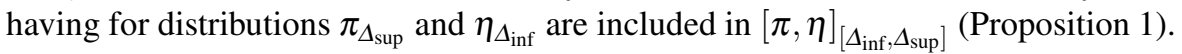

Let us denote $F_{\pi}^{-}, F_{\pi}^{+}$and $F_{\eta}^{-}, F_{\eta}^{+}$the cumulative distributions given by Eq. (5) respectively applied to the thin cloudy kernels $\pi_{\Delta_{\text {sup }}}$ and $\eta_{\Delta_{\text {inf }}}$. By Proposition 2 , they are

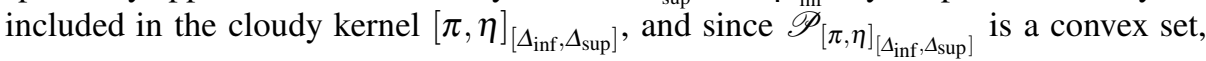
$1 / 2 F_{\pi}^{-}+1 / 2 F_{\pi}^{+}$and $1 / 2 F_{\eta}^{-}+1 / 2 F_{\eta}^{+}$are also included in the kernel. These two convex mixtures being equals to $F_{\Delta_{\text {inf }}}, F_{\Delta_{\text {sup }}}$, this ends the proof.

Proposition 4. The cloudy kernel $[\pi, \eta]_{\left[\Delta_{\mathrm{inf}}, \Delta_{\mathrm{sup}}\right]}$ includes any summative kernel $\kappa_{\Delta}$ having $F_{\Delta}$ for cumulative distribution with $\Delta \in\left[\Delta_{\text {inf }}, \Delta_{\text {sup }}\right]$.

Proof. We know, by Proposition 2 , that the thin cloudy kernel $[\pi, \eta]_{F_{\Delta}}$ such that

$$
\pi_{\Delta}(x)=\left\{\begin{array}{cc}
2 * F_{\Delta} & \text { if } x \leq 0 \\
2 *\left(1-F_{\Delta}\right) & \text { if } x \geq 0
\end{array}\right.
$$

includes the cumulative distribution $[\pi, \eta]_{F_{\Delta}}$. Also, we have that $F_{\Delta_{\text {inf }}}(x) \leq F_{\Delta}(x) \leq$ $F_{\Delta_{\text {sup }}}(x)$ for $x \leq 0$, and $F_{\Delta_{\text {sup }}}(x) \leq F_{\Delta}(x) \leq F_{\Delta_{\text {inf }}}(x)$ for $x \geq 0$, due to the symmetry of the retained summative kernels. This means that $\pi_{\Delta_{\text {sup }}} \leq \pi_{\Delta} \leq \eta_{\Delta_{\text {inf }}}$, therefore the thin cloudy kernel $[\pi, \eta]_{F_{\Delta}}$ is included in $[\pi, \eta]_{\left[\Delta_{\text {inf }}, \Delta_{\text {sup }}\right.}$, and this ends the proof. 
Let us now show that the proposed cloudy kernels exclude summative kernels with a bandwidth smaller than $\Delta_{\text {inf }}$, among which is the Dirac measure.

Proposition 5. Any kernel $\kappa_{\Delta}$ having $F_{\Delta}$ for cumulative distribution with $\Delta \leq \Delta_{\text {inf }}$ or $\Delta \geq \Delta_{\text {sup }}$ is not included in the cloudy kernel $[\pi, \eta]_{\left[\Delta_{\text {inf }}, \Delta_{\text {sup }}\right]}$

Proof. Similar to the one of Proposition 4 , considering that the thin cloud induced by $F_{\Delta}$ when $\Delta \leq \Delta_{\text {inf }}$ is not included in the cloudy kernel $[\pi, \eta]_{\left[\Delta_{\text {inf }}, \Delta_{\text {sup }}\right]}$.

These proposition show that cloudy kernels are fitted to our purpose, i.e., representing sets of summative kernels with lower- and upper-bounded bandwidth. Still, as for maxitive kernels, other kernels than the summative kernels belonging to the family $\kappa_{\Delta}$ are included in $\mathscr{P}_{[\pi, \eta]_{\left[\Delta_{\text {inf }}, \Delta_{\text {sup }}\right]}}$.

\section{Practical Computations}

In practice, imprecise filtering is done by extending the expectation operator to representations inducing probability sets, in our case by using Choquet integrals [9]. In this section, we recall what is a Choquet integral and its links with expectation operators. We then propose an efficient algorithm to compute this Choquet integral for cloudy kernels. To shorten notations $[\pi, \eta]_{\left[\Delta_{\text {inf }}, \Delta_{\text {sup }}\right]}, \eta_{\Delta_{\text {inf }}}$ and $\pi_{\Delta_{\text {sup }}}$ will be denoted by $[\pi, \eta], \eta$ and $\pi$. Since computations are achieved on a discretised space, we consider that we are working on a finite domain $\mathscr{X}$ of $N$ elements. In our case, this space corresponds to a finite sampling of the signal.

\subsection{Expectation Operator and Choquet Integral}

Consider the domain $\mathscr{X}=\left\{x_{1}, \ldots, x_{N}\right\}$ with an arbitrary indexing of elements $x_{i}$ (not necessarily the usual ordering between real numbers) and a real-valued function $f$ (here, the sampled values of the signal) on $\mathscr{X}$, together with a discretized summative kernel $\kappa_{i}, i=1, \ldots, N$, where $\kappa_{i}=\kappa\left(x_{i}\right)$. Classical convolution between the kernel $\kappa$ and the sampled signal $f$ is equivalent to compute the expectation $\mathbb{E}_{\kappa}(f)=\sum_{i=1}^{N} \kappa_{i} f\left(x_{i}\right)$.

When working with a set $\mathscr{P}$ of summative kernels defined on $\mathscr{X}$, the expectation operator $\mathbb{E}(f)$ becomes inter-valued $[\underline{\mathbb{E}}(f), \overline{\mathbb{E}}(f)]$, with $\underline{\mathbb{E}}(f)=\inf _{\kappa \in \mathscr{P}} \mathbb{E}_{\kappa}(f)$ and $\overline{\mathbb{E}}(f)=\sup _{\kappa \in \mathscr{P}} \mathbb{E}_{\kappa}(f)$. These bounds are generally hard to compute, still there are cases where practical tools exist that make their computation more tractable. First recall [10] that lower and upper confidence measures of $\mathscr{P}$ on an event $A \subseteq \mathscr{X}$ are such that $\underline{P}(A)=\inf _{\kappa \in \mathscr{P}} P_{\kappa}(A)$ and $\bar{P}(A)=\sup _{\kappa \in \mathscr{P}} P_{\kappa}(A)$ and are dual in the sense that $\underline{P}(A)=1-\bar{P}\left(A^{c}\right)$. If $\underline{P}$ satisfy a property of 2 -monotonicity, that is if for any pair $\{A, B\} \subseteq \mathscr{X}$ we have $\underline{P}(A \cap B)+\underline{P}(A \cup B) \geq \underline{P}(A)+\underline{P}(B)$, then expectation bounds can be computed by a Choquet Integral.

Consider a positive bounded function $2 f$ on $\mathscr{X}$. If we denote by () a reordering of elements of $\mathscr{X}$ such that $f\left(x_{(1)}\right) \leq \ldots \leq f\left(x_{(N)}\right)$, the Choquet Integral giving the lower expectation reads

${ }^{2}$ Positivity is not constraining here, since if $c$ is a constant $\underline{\mathbb{E}}(f+c)=\underline{\mathbb{E}}(f)+c$ and the same holds for $\overline{\mathbb{E}}$. 


$$
C_{\underline{P}}(f)=\underline{\mathbb{E}}(f)=\sum_{i=1}^{N}\left(f\left(x_{(i)}\right)-f\left(x_{(i-1)}\right) \underline{P}\left(A_{(i)}\right),\right.
$$

with $f\left(x_{(0)}\right)=0$ and $A_{(i)}=\left\{x_{(i)}, \ldots, x_{(N)}\right\}$. Upper expectation can be computed by replacing the lower measure $\underline{P}$ by the upper one $\bar{P}$. The main difficulty to evaluate Eq. (7) is then to compute the lower (or upper) confidence measure for the $N$ sets $A_{i}$.

\subsection{Imprecise Expectation with Cloudy Kernels}

Cloudy kernels satisfying Definition 2 induce lower confidence measure that are $\infty$ monotone [8]6], hence Choquet integral can be used to compute lower and upper expectations. Let us now detail how the lower confidence measure value on events can be computed efficiently (upper confidence measure are obtained by duality). Cloudy kernels $[\pi, \eta]$ defined on $\mathscr{X}$ induce a complete pre-order $\leq_{[\pi, \eta]}$ between elements of $\mathscr{X}$, in the sense that $x \leq_{[\pi, \eta]} y$ if and only if $\eta(x) \leq \eta(y)$ and $\pi(x) \leq \pi(y)$. Given a set $A \subseteq \mathscr{X}$, we denote respectively by $\underline{x}_{A}$ and by $\bar{x}_{A}$ its lowest and highest elements with respect to $\leq_{[\pi, \eta]}$. We now introduce the concepts of $[\pi, \eta]$-connected sets, that are instrumental in the computation of lower confidence measures.

Definition 4. Given a cloudy kernel $[\pi, \eta]$ over $\mathscr{X}$, a subset $C \subseteq \mathscr{X}$ is $[\pi, \eta]$-connected if it contains all elements between $\underline{x}_{C}$ and by $\bar{x}_{C}$, that is $C=\left\{x \in \mathscr{X} \mid \underline{x}_{C} \leq_{[\pi, \eta]} x \leq_{[\pi, \eta]} \bar{x}_{C}\right\}$

We denote by $\mathscr{C}$ the set of all $[\pi, \eta]$-connected sets of $\mathscr{X}$. Now, any event $A$ can be inner approximated by another event $A_{*}$ such that $A_{*}=\bigcup_{C \in \mathscr{C}, C \subset A} . C$ is the union of all maximal $[\pi, \eta]$-connected sets included in $A$. Due to an additivity property of the lower confidence measure on $[\pi, \eta]$-connected sets [11], $\underline{P}(A)$ is then

$$
\underline{P}(A)=\underline{P}\left(A_{*}\right)=\sum_{C \in \mathscr{C}, C \subset A} \underline{P}(C)
$$

We consider that elements of $\mathscr{X}$ are indexed accordingly to $\leq_{[\pi, \eta]}$, i.e., elements $x_{1}, \ldots$, $x_{N}$ are indexed such that $i \leq j$ if and only if $\eta\left(x_{i}\right) \leq \eta\left(x_{j}\right)$ or $\pi\left(x_{i}\right) \leq \pi\left(x_{j}\right)$. Given this ordering, the lower confidence measure of a $[\pi, \eta]$-connected set $C=\left\{x_{i}, \ldots, x_{j}\right\}$ is given by the simple formula

$$
\underline{P}(C)=\max \left\{0, \eta\left(x_{j+1}\right)-\pi\left(x_{i-1}\right)\right\},
$$

with $\eta\left(x_{N+1}\right)=1$ and $\pi\left(x_{0}\right)=0$. Note that, as $\leq_{[\pi, \eta]}$ is a pre-order, we have to be cautious about equalities between some elements. Figure 2 illustrates a cloudy kernel with 7 (irregularly) sampled values, its associated indexing and order.

Algorithm 1 describes how to compute lower confidence measures and the incremental summation giving the lower expectation. At each step, the $[\pi, \eta]$-connected sets forming $A_{(i)}$ are extracted and the corresponding lower confidence measure is computed. The value of the Choquet integral is then incremented. To simplify the algorithm, we assume $\leq_{[\pi, \eta]}$ to be an order (i.e., it is asymmetric). Note that two orderings and indexing are used in the algorithm: the one where elements are ordered by values of $f$, denoted by (), and the other where elements are ordered using $\leq_{[\pi, \eta]}$, without parenthesis. Except if the function $f$ is increasingly monotonic in $\mathbb{R}$, the indexing following the natural order of numbers is never used. 


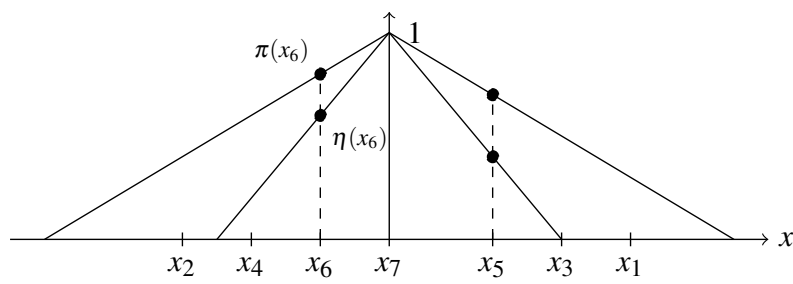

$x_{1} \leq_{[\pi, \eta]} x_{2} \leq_{[\pi, \eta]} x_{3} \leq_{[\pi, \eta]} x_{4} \leq_{[\pi, \eta]} x_{5} \leq_{[\pi, \eta]} x_{6} \leq_{[\pi, \eta]} x_{7}$

Fig. 2. Discretization of cloudy kernels and indexing of elements around $x_{7}$ (each $x_{i}$ corresponds to a sampled value)

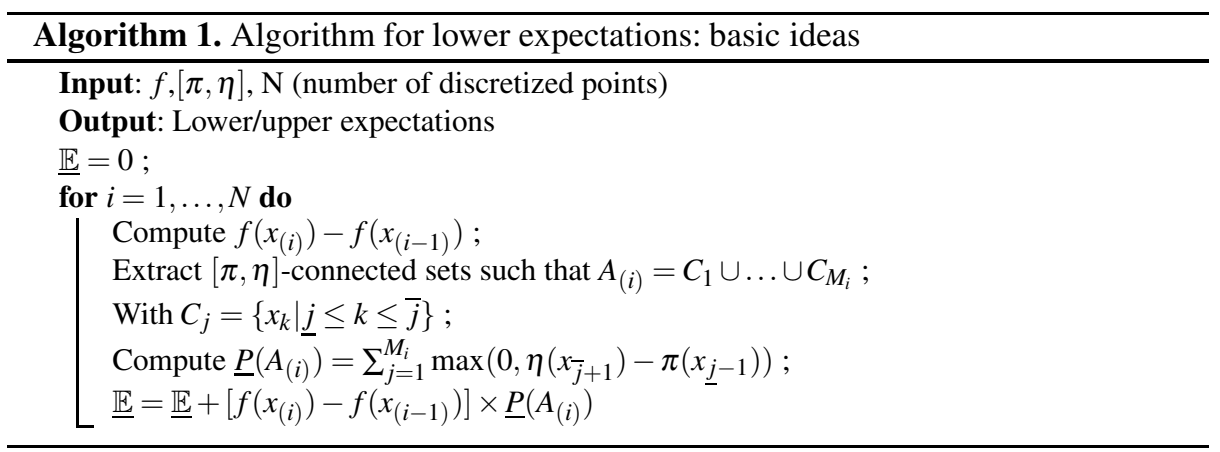

\section{Experiment: Comparison with Summative and Maxitive Kernels}

Let us now illustrate the advantage of using cloudy kernels rather than simple maxitive kernels when filtering a noisy signal. Figure 3 shows in cyan a (noisy) signal that has to be filtered by a smoothing kernel. Imprecise kernels (cloudy or maxitive) can be used if one does not know the exact shape of the impulse response of the filter, but can assume that this filter is symmetric, centred and has a lower and upper bounded bandwidth $\Delta \in\left[\Delta_{\text {inf }}, \Delta_{\text {sup }}\right]$. The signal pictured in 3 has been obtained by summing nine sine waves with random frequencies and then by adding a normal centered noise with a standard deviation $\sigma=5$.

Assume that the summative kernels to be considered are the uniform ones bounded by $\Delta \in[0.018,0.020]$. The most specific maxitive kernel dominating this family is the triangular kernel with a bandwidth equal to 0.02 (see [2]). The bounds obtained by using such a kernel are displayed on Figure 3 (dotted red and blue lines). As expected, the inclusion of the Dirac measure in the maxitive kernel gives very large upper and lower filtered bounds, that encompass the whole signal (i.e. the signal is always in the interval provided by the maxitive kernel). Given our knowledge about the desired bandwidth, it is clearly desirable to also take account of the lower bound 0.018 . 


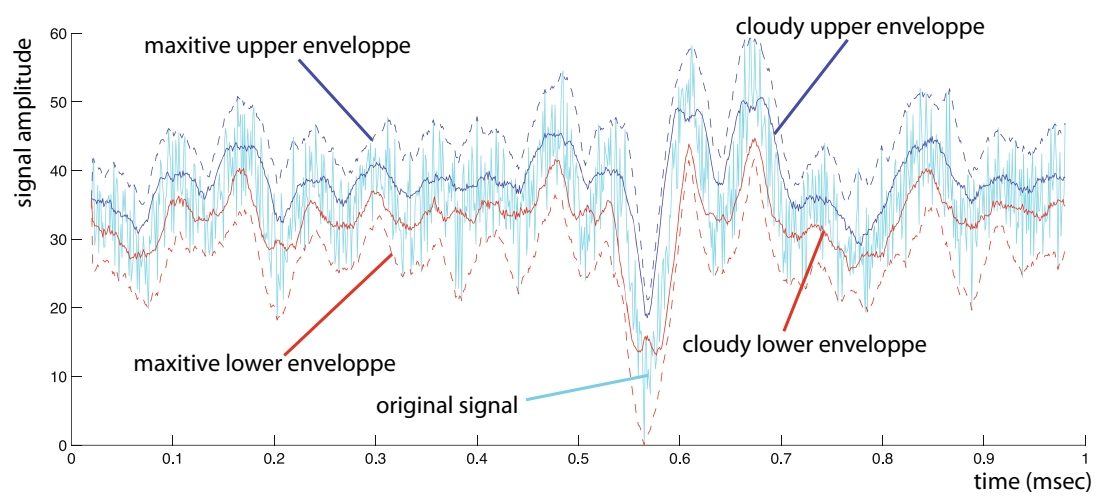

Fig. 3. Superposition of the original signal (cyan), the maxitive imprecise filtering (dotted blue upper, dotted red - lower) and the cloud based imprecise filtering (blue - upper, red - lower)

Cloudy kernels can model a more specific set of summative kernels, accounting for the lower bound, by using the cloudy kernel composed of two triangular maxitive kernels, the lower kernel having a bandwidth $\Delta_{\text {inf }}=0.018$ and the upper kernel having a bandwidth $\Delta_{\text {sup }}=0.020$, and filtering the signal with Algorithm 1 The result is also pictured in Figure 3 (full red and blue lines). We can see that the lower and upper bounds are now much tighter, as expected. Hence, we now have bounds to whose are associated a good confidence and that are more informative.

To illustrate the capacity of maxitive and cloudy kernels to encompass the desired kernels, we have plotted on 4 ten filtered signals (in cyan) obtained by using different symmetric centered summative kernels whose bandwidth belongs to the interval $\left[\Delta_{\text {inf }}, \Delta_{\text {sup }}\right]$. Every filtered signal belongs to the interval-valued signal obtained by using the cloudy kernel based approach.

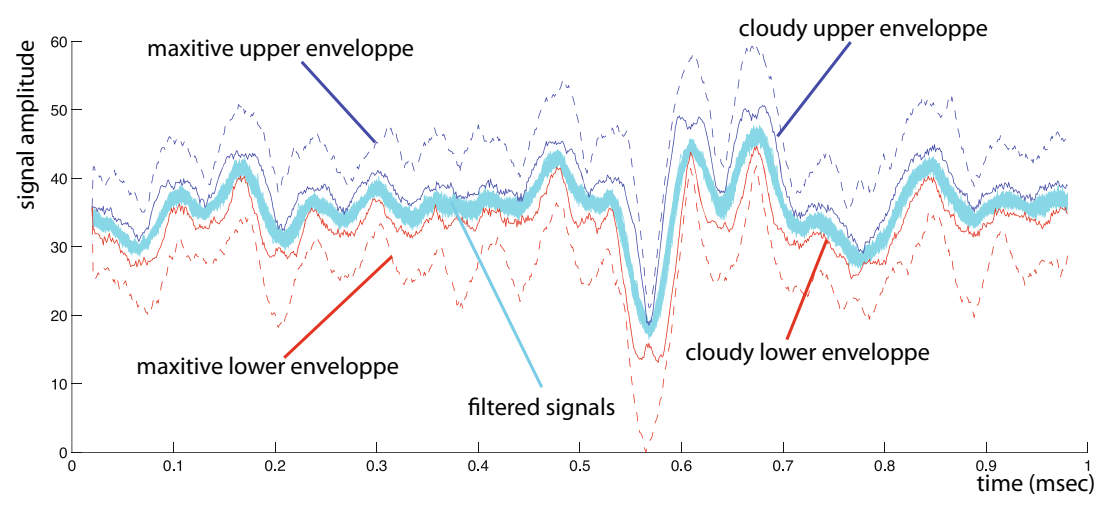

Fig. 4. Superposition of nine filtered signals (cyan), the maxitive imprecise filtering (dotted blue upper, dotted red - lower) and the cloud based imprecise filtering (blue - upper, red - lower) 


\section{Conclusion}

Both summative and maxitive kernels suffer from some defects when it comes to filter a given signal. The former asks for too much information and the latter is often too imprecise to give tight information. In this paper, we have proposed to use cloudy kernels (using the uncertainty representations called cloud) as a compromis between the two representations to achieve imprecise linear filtering. We have also proposed a simple and efficient (but not necessarily the most efficient) algorithm to compute lower and upper bounds of the filtered signal.

Our experiments show that using cloudy kernels does have the expected properties. Compared to summative and maxitive kernels, they allow to retrieve reliable and informative envelope for the filtered signal. However, it appears that envelopes resulting from the filtering using cloudy kernel are still not so smooth. We suspect that this is due to summative kernels inside the cloudy kernels for which probability masses are concentrated around some particular points (i.e. mixtures of Dirac measures). To avoid this, we could consider the use of technics already proposed [12] to limit the accumulation of such probability masses.

\section{References}

1. Jan, J.: Digital Signal Filtering, Analyses and Restoration. IET (2000)

2. Loquin, K., Strauss, O.: On the granularity of summative kernels. Fuzzy Sets and Systems 159(1952-1972) (2008)

3. Dubois, D., Prade, H.: Possibility Theory: An Approach to Computerized Processing of Uncertainty. Plenum Press, New York (1988)

4. Dubois, D., Prade, H.: When upper probabilities are possibility measures. Fuzzy Sets and Systems 49, 65-74 (1992)

5. Neumaier, A.: Clouds, fuzzy sets and probability intervals. Reliable Computing 10, 249-272 (2004)

6. Destercke, S., Dubois, D., Chojnacki, E.: Unifying practical uncertainty representations: II clouds. Int. J. of Approximate Reasoning (in press, 2007)

7. Baudrit, C., Dubois, D.: Practical representations of incomplete probabilistic knowledge. Computational Statistics and Data Analysis 51(1), 86-108 (2006)

8. Destercke, S., Dubois, D., Chojnacki, E.: Unifying practical uncertainty representations: I generalized p-boxes. Int. J. of Approximate Reasoning (in press, 2008)

9. Denneberg, D.: Non-additive measure and integral, basic concepts and their role for applications. In: Fuzzy Measures and Integrals - Theory and Applications, pp. 42-69. Physica Verlag, Heidelberg (2000)

10. Walley, P.: Statistical reasoning with imprecise Probabilities. Chapman and Hall, New York (1991)

11. Destercke, S., Dubois, D.: The role of generalised p-boxes in imprecise probability models. In: Augustin, T., Coolen, F., Moral, S., Troffaes, M.C.M. (eds.) Proc. of the 6th Int. Symp. on Imprecise Probability: Theories and Applications, pp. 179-188 (2009)

12. Kozine, I., Krymsky, V.: Enhancement of natural extension. In: Proc. 5th Int. Symp. on Imprecise Probabilities: Theories and Applications (2007) 